\title{
An adverse reaction after hyaluronic acid filler application: a case report
}

\author{
Maša Vidič ${ }^{1}$, Igor Bartenjev²凶
}

\begin{abstract}
Among several skin fillers developed in recent decades, hyaluronic acid (HA) fillers have become the material of choice. They are safe, long-lasting, not immunogenic, and cost effective, and they can be removed with hyaluronidase. Unfortunately, early and delayed complications can also occur following HA filler injection. Here we report the case of a hyaluronic filler-related complication in a 50-year-old female patient. The locations affected were the forehead, glabella, and nose. Three days after HA filler application in the upper lip, glabellar region, and nasal root, with no immediate diverse reaction at the time of application, an erythematous, livedoid rash with a well-defined border occurred at the site of the injection in the glabellar region, including the area from the nasal root to the scalp and left upper eyelid. Because an infection or allergy was suspected, she was first given an antibiotic for 6 days p.o. and later also a corticosteroid systemically with good results. Two years later (in 2017) the patient decided to repeat the treatment with $\mathrm{HA}$ injections in the glabellar region and experienced no adverse reactions.
\end{abstract}

Keywords: fillers, hyaluronic acid, adverse reaction, glabellar region

Received: 6 April 2018| Returned for modification: 7 May 2018 | Accepted: 7 May 2018

\section{Introduction}

Several skin fillers have been developed in recent decades for correcting signs of facial aging such as wrinkles and reducing lip volume $(1,2)$. These fillers are bovine and human collagen, hyaluronic acid (HA), calcium hydroxyl apatite, poly-L-lactic acid, silicone, and other formulations (3). Starting with bovine collagen injections in the 1980s, researchers have been searching for the ideal skin filler, which should be safe, effective, biocompatible, non-immunogenic, easy to remove if necessary, and inexpensive, require no allergy testing, have an acceptable persistency, and be easy to distribute and store. Hyaluronic acid dermal fillers have most of these features $(2,4)$.

$\mathrm{HA}$ is the dominant material for use in cosmetic skin correction and has specific advantages over other fillers: it is longer lasting, less immunogenic, and more convenient than other softtissue fillers $(2,5)$. It is a glycosaminoglycan, one of the body's own substances in the form of a linear polysaccharide consisting of repeating units of glucuronic acid and $\mathrm{N}$-acetyl-glucosamine found in the extracellular matrix of connective tissue, synovial fluid, hyaline cartilage, the vitreous body of the eye, disc nuclei, the umbilical cord, joints, muscles, and skin dermis $(2,4)$. It is a naturally occurring ubiquitous component in the human body, which, when combined with water, forms a viscous gel $(2,4,6)$. It is responsible for drawing water into the skin, giving it volume while binding collagen and elastin fibers into a supportive matrix that gives the skin its structure (4).

HA has no tissue or species specificity. It has the same structure whether it derives from bacteria, animals, or humans, and thus has a low potential for allergic or immunogenic reactions $(2,4)$. Unmodified HA has a half-life of approximately 12 hours before it is rapidly broken down by an enzyme called hyaluronidase. Because hyaluronidase acts at the site of local injection and hydrolyses HA rapidly, HA fillers can easily be removed from the concerned area (4). For use as dermal filler, only a few hours of longevity are insufficient and a more durable material is preferred. As such, researchers have altered the chemistry of HA by cross-linking chains to create a larger, more stable molecule with longer residue time in tissues (approximately a few months), similar biocompatibility, and viscoelastic filling properties $(2,7)$.

\section{Case report}

We present the case of a healthy 50-year-old woman that received injections of HA several times at our outpatient department and once experienced an unwanted reaction of unclear origin after application in the glabellar region. The first time she was treated with HA injection in the glabellar region and nasolabial fold with no adverse reaction was in 2014. A year later (in 2015), she repeated the same treatment, this time in the upper lip, glabellar region, and nasal root with no diverse reaction at time of application. After approximately 72 hours an erythematous, livedoid rash with a well-defined border occurred at the site of the injection in the glabellar region, including the area from the nasal root to the scalp and left upper eyelid (Fig. 1). The patient's eyelids were swollen, but she experienced no pain or itching. No rash was seen at other sites of injection (e.g., the upper lip) and no enlarged adjacent lymph nodes were noticed. Because an infection was suspected, she was first given an antibiotic (azithromycin 500 $\mathrm{mg}$ ) for 6 days p.o. and later also a corticosteroid (methylprednisolone $32 \mathrm{mg}$ ) p.o. for 5 days. In 10 days the rash improved, and it disappeared completely a month after the application of HA (Fig. 2). Three years later (in 2017), despite our concern, the patient decided to repeat the treatment with HA injections in the glabellar region and experienced no adverse reactions after application. What happened after the second application of HA filler?

\section{Discussion}

\section{Anatomic specificity of the glabellar region}

Certain areas of the face demand special attentiveness because of their specific arterial anatomy. An example is the glabellar region, with the blood supply provided by small vessels branching from 
the supratrochlear and supraorbital arteries with limited collateral circulation (8). If these vessels are damaged and blood supply is lost-which is possible while injecting dermal fillers in this area owing to the superficial location and vicinity of the smaller branched vessels to the glabellar crease-tissue viability may be compromised (8).

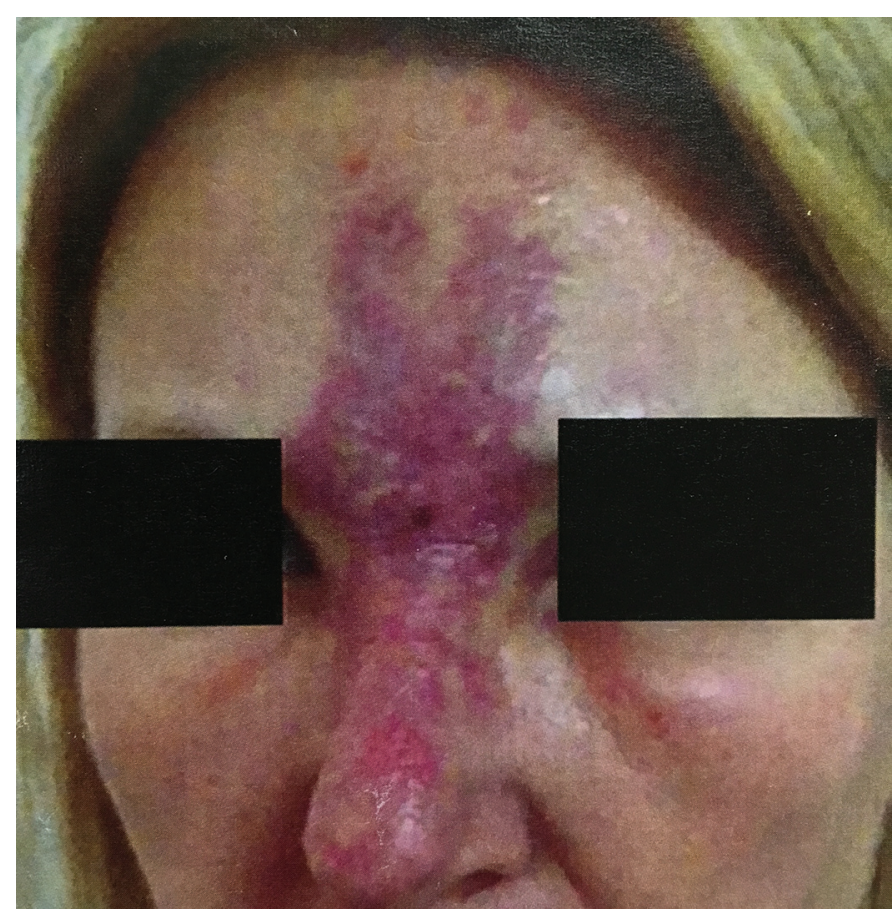

Figure 1 | Erythematous, livedoid rash (3 days after injection of hyaluronic acid).

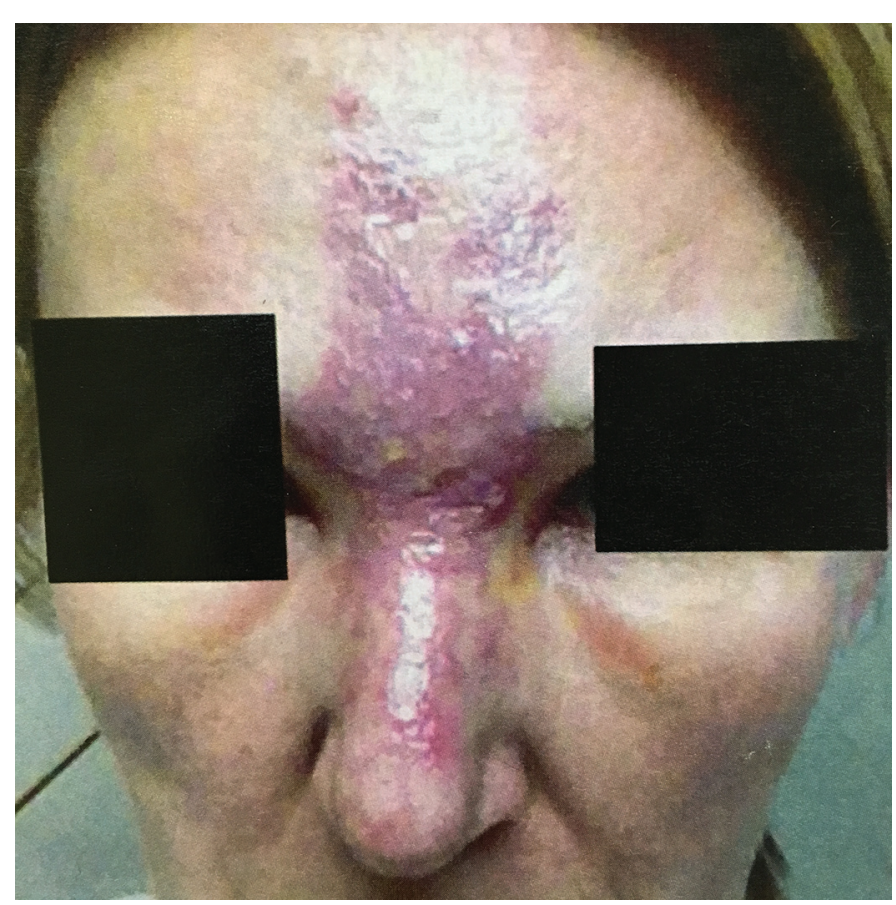

Figure 2 | Improvement (14 days after application).

\section{Complications of injectable dermal fillers}

Mild pain, erythema, swelling, and bruising at the injection site are common findings after dermal fillers applications. When performed correctly, HA injections are associated with a very low rate of adverse reaction and rarely result in a more serious complication $(9,10)$. Nevertheless, these can arise, ranging from minor to severe complications requiring prompt treatment (10).

Early reactions include erythema, bruising, bleeding, pain, in- fections, allergic reactions, vascular infarction, soft-tissue necrosis, inappropriate placement, and distant spread. Late reactions include infections, formation of nodules and granulomas, dyspigmentation, and displacement of HA filler material (10).

\section{Vascular infarction and skin necrosis}

Skin necrosis is a rare but potentially devastating complication of treatment with injectable fillers. It is a consequence of untreated vascular compromise, which can result from either arterial or venous obstruction. Possible causes are direct trauma to the vessel wall, inadvertent intravascular injection of injectable fillers, or direct pressure of the filling material on the vessel, causing obstruction of the lumen. Injection-related edema is another possible mechanism of compromised blood flow by contributing an external force on the vessel wall (9). The initial signs and symptoms (although not pathognomonic) of impaired blood flow include immediate pain, pallor or blanching, blotchy reddish or livedoid discoloration, slow capillary refill, blue or gray-blue discoloration, and demarcation with a distinct margin of hyperemia surrounding frank necrosis, followed by skin slough, tissue repair, and remodeling if the occlusion persists (11). Impending necrosis may resolve without permanent damage. No signs are sometimes noted at the time of injection because delayed compression of vessels by the product is one of the possible mechanisms of injury (12). If an artery is occluded, immediate and severe pain and blanching occur, whereas venous obstruction usually manifests with a delayed reticulated, violaceous appearance (9).

\section{Visual impairment}

A devastating complication of intravascular injection of a filler substance is blindness (10). If a bolus is injected into the glabellar region, the needle might enter the supraorbital artery. The pressure at the end of the needle causes the product to flow retrograde into the ophthalmic artery and from there possibly to the small central retinal artery. There the bolus can lodge in the retina and cause vision loss. Patients present with a sudden blind spot or visual field deficit, which is practically irreversible (11).

\section{Allergic reactions (hypersensitivity)}

Hypersensitivity is an exaggerated immune reaction of the body to a foreign substance. Such reactions usually occur within minutes of exposure to an antigen. Histamine release causes increased vascular permeability, edema, erythema, pain, and itching. Delayed hypersensitivity reactions are also possible, characterized by erythema, edema, itching, and a change in the contour of the injected implant, and, occasionally, an indurated papule or inflamed dermal nodule. Allergic reactions following HA injections, especially severe cases with rapidly progressing angioedema, are very rare $(10,13)$.

\section{Infections}

Although uncommon, cellulitis, abscess formation, and other infectious complications may occur when applying injectable fillers in the skin because its barrier function is temporarily disrupted. It can be caused by bacterial, viral, or fungal species, or as a polymicrobial infection $(7,9,13)$. Bacterial infections are usually caused by common skin and soft tissue pathogens, such as Staphylococ- 
cus aureus. Clinical signs are erythema, swelling, pain, and single or multiple erythematous and/or fluctuant nodules, whereas presentation of a new lesion more than 2 weeks after the procedure suggests an atypical infection, usually with mycobacteria (the lesion is often a firm, mildly tender mass or nodule with or without fluid). A systemic reaction such as fever, leukocytosis, weight loss, and fatigue may also be present (13). The most common viral infection after injection is herpes simplex. The initial presentation is clear vesicles in the skin, but they may not always be evident (7).

\section{Nodules and granulomas}

Skin nodules are a known complication of injectable fillers. Nonerythematous nodules form immediately after the injection when too much product is applied into a small area $(7,9,13)$. Such nodules are different from the inflammatory reactions that occur early after injection, whether as a reaction to injury or infection (13). Infection presents as single or multiple nodules with inflammatory signs; they appear subcutaneously or in the dermis and may be painful or not. Sometimes fibrotic nodules occur that are usually painless, palpable, and mostly invisible. They may be a consequence of a fibrous reaction as a response to the presence of the product or transient inflammatory granulomatous reactions, when foreign body reactions precipitate the appearance of lumps leading to granuloma formation $(7,9,13)$.

\section{Skin discolorations}

Skin discoloration can occur at the site of injection, usually appearing immediately after application and generally resolving within a few weeks. Erythema occurs as a result of inflammation, whiteness can be a sign of overcorrection with the injected substance, and hyperpigmentation and bluish discoloration sometimes follow injections with HA. Bluish skin can represent traces of hemosiderin associated with vascular injury and visual distor- tion from light refraction to the filler through the skin (the Tyndall effect) (13). This is caused by an injection of HA filler too close to the surface of the skin. Melanin in the dermis displays a blue tint, or "Mongolian spots." The Tyndall effect does not change over time, and it remains until the material is removed using hyaluronidase injection (13).

\section{Conclusion}

This article reported a rare case of complication after HA filler application in the glabellar region. It is also a case for discussion of what happened at a second application of HA filler. In general, HA injections are associated with a very low rate of adverse reaction or serious complications $(9,10)$. In our case, the result of the application was not skin necrosis, which is a possible consequence of untreated vascular compromise. There were no clinical signs of impaired blood flow, which include immediate pain, pallor, or blanching (11). Delayed hypersensitivity reactions are also possible, characterized by erythema, edema, itching, a change in the contour of the injected implant, and, occasionally, an indurated papule or inflamed dermal nodule, but they are very rare after HA application. In our case, the reaction was limited to the glabellar area only. Two years later, a repeated application of HA filler (the same substance, the same producer, and the same area) was not followed by complications of any kind, which makes the theory of a late allergic reaction unlikely. Clinical signs of infection are erythema, swelling, pain, and single or multiple erythematous and/or fluctuant nodules. Although uncommon, cellulitis, abscess formation, and other infectious complications may occur when applying injectable fillers in the skin $(7,9,13)$. Our patient experienced no pain or itching. No rash was seen at other sites of injection (upper lip) and no enlarged adjacent lymph nodes were noticed, but this would be expected in the case of a severe regional bacterial infection. We believe that a bacterial infection is the most probable cause of the atypical complication in our patient, but doubt remains and the matter is not settled.

\section{References}

1. Lowe NJ, Maxwell CA, Lowe P, Duick MG, Shah K. Hyaluronic acid skin fillers: adverse reactions and skin testing. J Am Acad Dermatol. 2001;45:930-3.

2. Monheit GD, Coleman KM. Hyaluronic acid fillers. Dermatol Ther. 2006;19:14150.

3. Cohen JL. Understanding, avoiding, and managing dermal filler complications. Dermatol Surg. 2008;34:92-9.

4. Brandt FS, Cazzaniga A. Hyaluronic acid gel fillers in the management of facial aging. Clin Interv Aging. 2008;3:153-9.

5. Park TH, Seo SW, Kim JK, Chang CH. Clinical experience with hyaluronic acidfiller complications. J Plast Reconstr Aesthet Surg. 2011;64:892-6.

6. Dayan SH. Complications from toxins and fillers in the dermatology clinic: recognition, prevention, and treatment. Facial Plast Surg Clin North Am. 2013;21: 663-73.

7. DeLorenzi C. Complications of injectable fillers, part I. Aesthet Surg J. 2013;33:n 561-75.
8. Glaich AS, Cohen JL, Goldberg LH. Injection necrosis of the glabella: protocol for prevention and treatment after use of dermal fillers. Dermatol Surg. 2006;32:276-81.

9. Daines SM, Williams EF. Complications associated with injectable soft-tissue fillers. A 5-year retrospective review. JAMA Facial Plast Surg. 2013;15:226-31.

10. Signorini M, Liew S, Sundaram H, De Boulle KL, Goodman GJ, Monheit G, et al. Global Aesthetics Consensus: avoidance and management of complications from hyaluronic acid fillers-evidence- and opinion-based review and consensus recommendations. Plast Reconstr Surg. 2016;137:961-71.

11. DeLorenzi C. Complications of injectable fillers, part 2: vascular complications. Aesthet Surg J. 2014:34:584-600.

12. Ozturk CN, Li Y, Tung R, Parker L, Piliang MP, Zins JE. Complications following injection of soft-tissue fillers. Aesthet Surg J. 2013;33:862-77.

13. Lowe NJ, Maxwell CA, Patnaik R. Adverse reactions to dermal fillers: review. Dermatol Surg. 2005;31:1616-25. 